\title{
Shocked flows induced by supersonic projectiles moving in tubes
}

\author{
Zonglin Jiang $^{\mathrm{a}, *}$, Yonghui Huang ${ }^{\mathrm{a}}$, Kazuyoshi Takayama ${ }^{\mathrm{b}}$ \\ ${ }^{a}$ LHD, Institute of Mechanics, Chinese Academy of Sciences, 15 Zhong Guan Cun Road, Beijing 100080, PR China \\ ${ }^{\mathrm{b}}$ SWRC, Institute of Fluid Science, Tohoku University, 2-1-1 Katahira, Aoba-ku, Sendai 980-8577, Japan
}

Received 25 July 2002; received in revised form 12 January 2003; accepted 19 March 2003

\begin{abstract}
A numerical study on shocked flows induced by a supersonic projectile moving in tubes is described in this paper. The dispersion-controlled scheme was adopted to solve the Euler equations implemented with moving boundary conditions. Four test cases were carried out in the present study: the first two cases are for validation of numerical algorithms and verification of moving boundary conditions, and the last two cases are for investigation into wave dynamic processes induced by the projectile moving at Mach numbers of $M_{p}=2.0$ and 2.4, respectively, in a short time duration after the projectile was released from a shock tube into a big chamber. It was found that complex shock phenomena exist in the shocked flow, resulting from shock-wave/projectile interaction, shock-wave focusing, shock-wave reflection and shock-wave/ contact-surface interactions, from which turbulence and vortices may be generated. This is a fundamental study on complex shock phenomena, and is also a useful investigation for understanding on shocked flows in the ram accelerator that may provide a highly efficient facility for launching hypersonic projectiles.

(C) 2003 Elsevier Ltd. All rights reserved.
\end{abstract}

\section{Introduction}

Shocked flows induced by a supersonic projectile moving from a shock tube into a big chamber are closely related to the ram accelerator research $[1,2,6,7]$. The problem is schematically shown in Fig. 1, where several wave phenomena are defined. When the projectile moves within the shock tube the diameter of which is set to be identical to the projectile's, the precursor shock wave is driven in front of the projectile, being similar to the piston/shock-tube problem. The precursor shock wave propagates into the big chamber first and develops into the first blast wave, which

\footnotetext{
* Corresponding author. Tel.: +86-10-625-459-47; fax: +86-10-625-570-81.

E-mail address: zljiang@imech.ac.cn (Z. Jiang).
} 


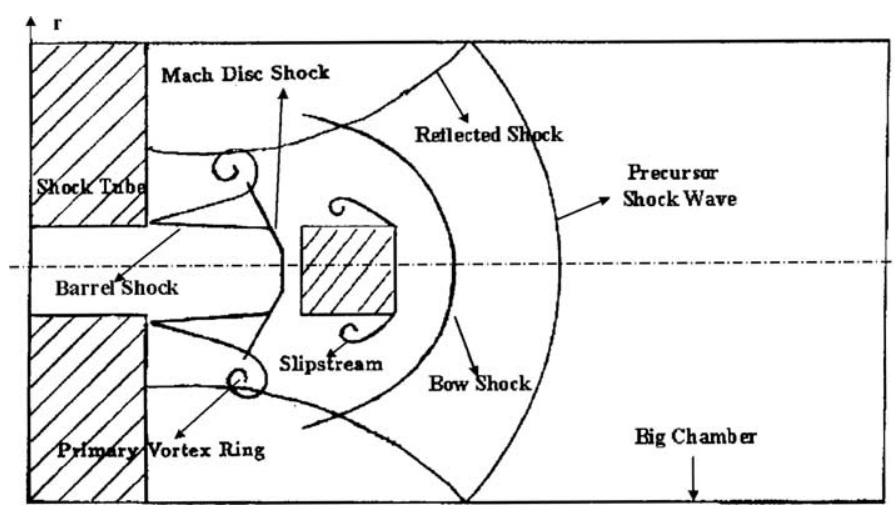

Fig. 1. Computational domain and phenomena definitions.

results in shock wave diffraction at the exit of the shock tube, shock wave reflection from the rigid wall of the big chamber, and a jet flow developing in the vicinity of the exit of the shock tube. Latter, when the projectile emerges from the shock tube and moves into the big chamber, the high pressure gas behind the projectile rushes out and the second blast wave develops. Moreover, once the projectile moves out of the supersonic flow region bounded by a barrel shock and a Mach disc, a bow shock wave is generated in front of the projectile. This bow shock wave can catch up with the precursor shock wave and will also interact with the shock waves reflected from the rigid wall. These result in the shock-wave/moving-body interaction, shock wave focusing and shock/contact surface interaction. This is an important topic for both shock wave research and engineering applications.

The relevant topic was referred to by Glass many years ago in his book entitled "Shock Waves and Man" [3], in which a series of photographs show the emergence of a 0.3-inch-diameter bullet from a rifle at 2200 feet per second $\left(M_{p}=2\right)$. An experimental study on axisymmetric supersonic flows around cylindrical projectiles has been presented by Matsumura et al. (1993) [4]. Their case is a supersonic projectile flying at a Mach number 4 in ambient air. Numerical work on shock wave flows generated by supersonic projectiles discharging from shock tubes into ambient air was reported by Jiang and Takayama [5] and in their work the interaction of the bow shock wave with the precursor shock wave was emphasized. Motivated by the need for hypersonic launching facilities, the ram accelerator has been intensively investigated both experimentally and numerically during the recent years $[1,2,6,7]$. However, most of the numerical researches on the ram accelerator were carried out by solving the governing equations with the Galilean transformation $[6,7]$, which may lead to some discrepancies between numerical results and experiments data, especially at the initial stage when the projectile is launched into the acceleration tube. The shocked flows containing many unsteady wave phenomena are still largely unexplored.

The present study aims at understanding on wave dynamic processes occurring in shocked flows at the projectile launching stage to demonstrates the unsteady flow phenomena and to provide useful information for the research on the ram accelerator and on the gun-firing problem $[8,14]$. The dispersion-controlled scheme based on the dispersion conditions $[9,10]$ was used for solving the Euler equations implemented with moving boundary conditions. These governing 
equations are chosen because the wave dynamic processes are emphasized in the present study, in which viscosity effects are negligible [15]. If the phenomena related to the turbulence and viscosity are to be examined, the Navier-Stokes equations with a suitable turbulence model have to be adopted. Two special cases were chosen for validation of numerical algorithms. The first one is the shock wave propagating from a shock tube into a big diameter chamber, which is the similar to the computational domain shown in Fig. 1, but without the projectile. The second case is a supersonic projectile flying in open space for verifying moving boundary conditions. After checking accuracy of the numerical algorithms, further two cases of the shocked flows induced by the supersonic projectile moving at two different Mach numbers were simulated and numerical results were presented in a time sequence of isobars and isopycnics. From numerical solutions, wave dynamics processes occurring in shocked flows were discussed in detail from a view point of shock wave dynamics.

\section{Governing equations}

Assuming that the shocked flow induced by supersonic projectiles in the present study is symmetrical during a short time duration of projectile launching and viscosity effects on wave dynamic processes are negligible, a hyperbolic system of conservation laws for the perfect gas in cylindrical coordinates can be written as

$$
\frac{\partial \mathbf{U}}{\partial t}+\frac{\partial \mathbf{F}}{\partial x}+\frac{\partial \mathbf{G}}{\partial r}+\frac{1}{r} \mathbf{S}=0
$$

where $\mathbf{U}, \mathbf{F}, \mathbf{G}$ and $\mathbf{S}$ denote the state variable, flux and source, respectively, given by

$$
\mathbf{U}=\left(\begin{array}{c}
\rho \\
\rho u \\
\rho v \\
e
\end{array}\right), \quad \mathbf{F}=\left(\begin{array}{c}
\rho u \\
\rho u^{2}+p \\
\rho u v \\
(e+p) u
\end{array}\right), \quad \mathbf{G}=\left(\begin{array}{c}
\rho v \\
\rho u v \\
\rho v^{2}+p \\
(e+p) v
\end{array}\right), \quad \mathbf{S}=\left(\begin{array}{c}
\rho v \\
\rho u v \\
\rho v^{2} \\
(e+p) v
\end{array}\right),
$$

where primitive variables in the unknown $\mathbf{U}$ are density $\rho$, velocity components $u$ and $v$, and total energy per unit volume $e$. Fluid pressure is denoted with $p$, which is related with the equation of state for the perfect gas given by

$$
e=\frac{p}{\gamma-1}+\frac{1}{2} \rho\left(u^{2}+v^{2}\right)
$$

where $\gamma$, the specific heat ratio, is taken to be 1.4 in the present study.

\section{Numerical methods}

The finite difference equations of (1a) discretized by using the dispersion-controlled scheme $[9,10]$ are given in the form of semi-discretion as 


$$
\left(\frac{\partial \mathbf{U}}{\partial t}\right)_{i, j}^{n}=-\frac{1}{\Delta x}\left(\mathbf{H}_{i+\frac{1}{2}, j}^{n}-\mathbf{H}_{i-\frac{1}{2}, j}^{n}\right)-\frac{1}{\Delta r}\left(\mathbf{P}_{i, j+\frac{1}{2}}^{n}-\mathbf{P}_{i, j-\frac{1}{2}}^{n}\right)-\frac{1}{r_{i, j}} \mathbf{S}_{i, j}^{n}
$$

where

$$
\left\{\begin{array}{l}
\mathbf{H}_{i+\frac{1}{2}, j}^{n}=\mathbf{F}_{i+\frac{1}{2} L, j}^{+}+\mathbf{F}_{i+\frac{1}{2} R, j}^{-} \\
\mathbf{P}_{i, j+\frac{1}{2}}^{n}=\mathbf{G}_{i, j+\frac{1}{2} L}^{+}+\mathbf{G}_{i, j+\frac{1}{2} R}^{-},
\end{array}\right.
$$

with

$$
\begin{aligned}
& \left\{\begin{array}{l}
\mathbf{F}_{i+\frac{1}{2} L, j}^{+}=\mathbf{F}_{i, j}^{+}+\frac{1}{2} \Phi_{A}^{+} \operatorname{minmod}\left(\Delta \mathbf{F}_{i-\frac{1}{2}, j}^{+}, \Delta \mathbf{F}_{i+\frac{1}{2}, j}^{+}\right), \\
\mathbf{F}_{i+\frac{1}{2} R, j}^{-}=\mathbf{F}_{i+1, j}^{-}-\frac{1}{2} \Phi_{A}^{-} \operatorname{minmod}\left(\Delta \mathbf{F}_{i+\frac{1}{2}, j}^{-}, \Delta \mathbf{F}_{i+\frac{3}{2}, j}^{-}\right),
\end{array}\right. \\
& \left\{\begin{array}{l}
\mathbf{G}_{i, j+\frac{1}{2} L}^{+}=\mathbf{G}_{i, j}^{+}+\frac{1}{2} \Phi_{B}^{+} \operatorname{minmod}\left(\Delta \mathbf{G}_{i, j-\frac{1}{2}}^{+}, \Delta \mathbf{G}_{i, j+\frac{1}{2}}^{+}\right), \\
\mathbf{G}_{i, j+\frac{1}{2} R}^{-}=\mathbf{G}_{i, j+1}^{-}-\frac{1}{2} \Phi_{B}^{-} \operatorname{minmod}\left(\Delta \mathbf{G}_{i, j+\frac{1}{2}}^{-}, \Delta \mathbf{G}_{i, j+\frac{3}{2}}^{-}\right),
\end{array}\right. \\
& \left\{\begin{array}{l}
\Delta \mathbf{F}_{i+\frac{1}{2}, j}^{ \pm}=\mathbf{F}_{i+1, j}^{ \pm}-\mathbf{F}_{i, j}^{ \pm}, \\
\Delta \mathbf{G}_{i, j+\frac{1}{2}}^{ \pm}=\mathbf{G}_{i, j+1}^{ \pm}-\mathbf{G}_{i, j}^{ \pm},
\end{array}\right. \\
& \left\{\begin{array}{l}
\mathbf{F}^{ \pm}=\mathbf{A}^{ \pm} \mathbf{U} \\
\mathbf{G}^{ \pm}=\mathbf{B}^{ \pm} \mathbf{U}
\end{array}\right. \\
& \left\{\begin{array}{l}
\boldsymbol{\Phi}_{A}^{ \pm}=\mathbf{I} \mp \beta \boldsymbol{\Lambda}_{A}^{ \pm}, \\
\boldsymbol{\Phi}_{B}^{ \pm}=\mathbf{I} \mp \beta \boldsymbol{\Lambda}_{B}^{ \pm},
\end{array}\right.
\end{aligned}
$$

where $\mathbf{A}$ and $\mathbf{B}$ are the Jacobian matrices of $\partial \mathbf{F} / \partial \mathbf{U}$ and $\partial \mathbf{G} / \partial \mathbf{U}$, respectively. $\mathbf{I}$ is a unit matrix, $\beta=\Delta t / \Delta x$, and $\boldsymbol{\Lambda}_{A}$ and $\boldsymbol{\Lambda}_{B}$ are vector matrices that consist of eigenvalues of matrices $\mathbf{A}$ and $\mathbf{B}$, respectively. In the above equations, the $(\cdot)^{+}$or $(\cdot)^{-}$superscript signs denote flux vector splitting according to the Steger and Warming method [11]. The time-marching integration was performed with a Runge-Kutta algorithms of second-order accuracy. As to the dispersion-controlled scheme, it has been constructed to meet the dispersion conditions [9,10], therefore, it is capable of capturing a discontinuity without any numerical oscillation and the need of additional artificial viscosity that may, otherwise, smear some physical phenomena. Such a characteristic is very helpful to highlight fine structures of shock wave interactions.

The computational geometry with sudden enlargement in its cross section is a shock tube connected to a big chamber, as schematically shown in Fig. 1. The shock tube is $70 \mathrm{~mm}$ long and $50 \mathrm{~mm}$ in diameter and the big chamber is $530 \mathrm{~mm}$ long and $200 \mathrm{~mm}$ in diameter. The projectile is $50 \mathrm{~mm}$ long and $50 \mathrm{~mm}$ in diameter. The inner diameter of the shock tube is chosen as a reference length in computation. The equally spaced grid system of $2400 \times 400$ mesh points was used along $x$ - and $r$-directions, respectively, and $280 \times 100$ points were distributed inside the shock tube. The computation was carried out only in the half of the computational domain because the flow field is 
assumed to be axisymmetric. Reflecting boundary conditions were specified both on solid walls and the axis of symmetry. Non-reflecting boundary conditions were applied at the inflow and outflow boundaries [13].

In order to simulate a moving projectile, two mesh systems are used in computation and both are equally spaced. The main mesh system is set in the laboratory-fixed coordinates and the moving mesh system is in the projectile-fixed ones. The moving mesh containing the projectile moves on the fixed main mesh at the projectile speed. The surfaces of the projectile are traced step by step so that the moving boundary conditions being consistent with the Euler equations and the numerical scheme could be applied on the projectile. The detailed descriptions of the moving boundary conditions has been reported by Jiang and Takayama [5]. The equations of the moving boundary conditions are given here for completeness.

$$
\left\{\begin{array}{l}
u_{a^{\prime}}=2.0 * U-u_{a}, \\
v_{a^{\prime}}=v_{a}, \\
\rho_{a^{\prime}}=\rho_{a}, \\
p_{a^{\prime}}=p_{a},
\end{array}\right.
$$

where $U$ is the projectile speed, a denotes the mesh point being adjacent to the projectile boundary and $\mathbf{a}^{\prime}$ is a's mirror image inside the projectile.

Oswatitsch [8] pointed that for high-speed projectiles, the most significant accelerations occur near the breech leaving the projectile speed relatively constant over most of the latter portion of its in-bore trajectory, the properties of the gas slug at the muzzle can be obtained by using the Rankine-Hugoniot relations under the assumption that the gas velocity is equal to the projectile launch speed. Schmit et al. [14] carried out their theoretical analysis based on the assumption and the obtained results agree well with experiments. Based on these pioneer works and considering the practical launching of a projectile into the acceleration tube, we simplify initial conditions for numerical simulations assuming that the friction between the projectile and the shock tube wall is negligible. In the initial stage, with the projectile moving down the shock tube, the precursor shock wave is taken as having arrived at the exit of the shock tube and the projectile is located at a distance being $110 \mathrm{~mm}$ behind the precursor shock wave. The surrounding condition in the big chamber is ambient air at $P_{\mathrm{a}}=1 \mathrm{~atm}$ and $T_{\mathrm{a}}=297 \mathrm{~K}$. Behind the precursor shock wave, the column of gas on either side of the projectile and the projectile itself all move at the same velocity, the so-called post-shock velocity, $V_{\mathrm{p}}$, determined according to a given Mach number of $M_{p}=V_{\mathrm{p}} / c$, where $c$ is referred to as the sound speed in ambient air. Using the projectile speed and the ambient air condition, the initial flow state between the precursor shock wave and the projectile can be specified with standard adiabatic shock relations [8]. The gas state behind the projectile is taken to be the same as the post-shock state in front of the projectile because that projectile moves loosely and the friction between the projectile and the shock tube wall is negligible.

\section{Accuracy check on numerical algorithms}

If flow physics is going to be explored with numerical solutions, checking accuracy on the numerical algorithms and validating numerical solutions are necessary. Two special cases were 
chosen for the purpose because experimental data for the present study are not available. The first case for validating numerical solutions is a shock wave propagating from a shock tube into a big chamber, being similar to the configuration as shown in Fig. 1 but without the projectile. The second one for verifying moving boundary conditions is a projectile flying in open space at a supersonic speed, being similar to the present study without the tubes. Accuracy of the present algorithms can be demonstrated in this way so that certain confidence could be gained on numerical simulations.

\subsection{Validation of numerical solutions with experiments}

In the first test case, the diameter ratio of the big chamber to the shock tube is taken to be $2: 1$. The initial shock Mach number of the transmitting shock wave is $M_{\mathrm{s}}=1.3$. The computational geometry is shown in Fig. 1, but without the projectile. The corresponding experiment was conducted with a specially designed aspheric cylindrical test section connected to the $55 \mathrm{~mm}$ diameter shock tube at the Shock Wave Research Center, Tohoku University, Japan. This test section permits collimated incident light rays to traverse the inner circular cross sectional bore parallel and emerge parallel. Interferometry is chosen to determine the density distribution within the flowfield because the density is more sensitive to small variations than other flow parameters, which can also provide qualitative information for CFD validation. For a direct comparison, the computational interferogram was constructed from numerical solutions by using techniques described by Jiang et al. [12]. Both the experimental interferogram and numerical result are presented in Fig. 2.

By comparing Fig. 2(a) with (b), it is obvious that agreement between the numerical result and the experiment is excellent. This is true not only because the number of fringes is identical but the
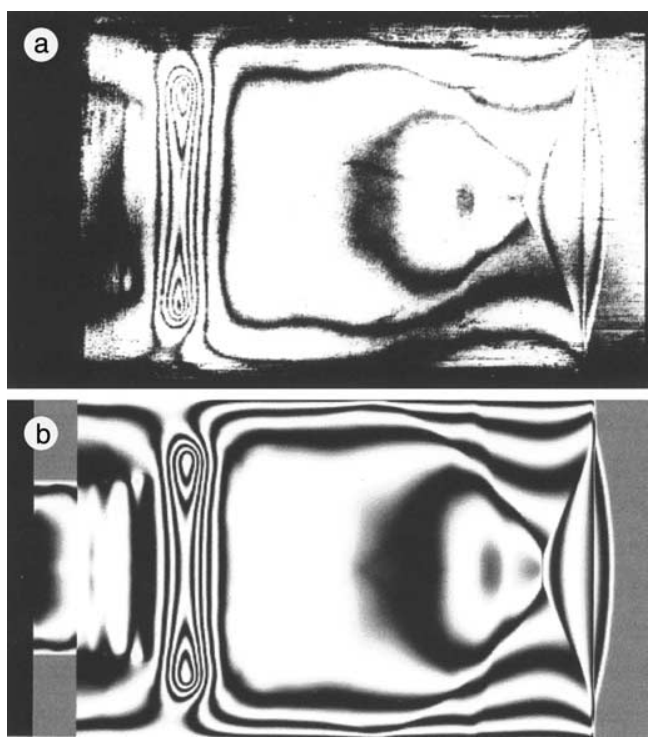

Fig. 2. Comparison of the experimental interferogram with the numerical one showing a transmitting shock wave propagating at $M_{\mathrm{s}}=1.3$ in a big chamber. (a) Experimental interferogram and (b) computational result. 


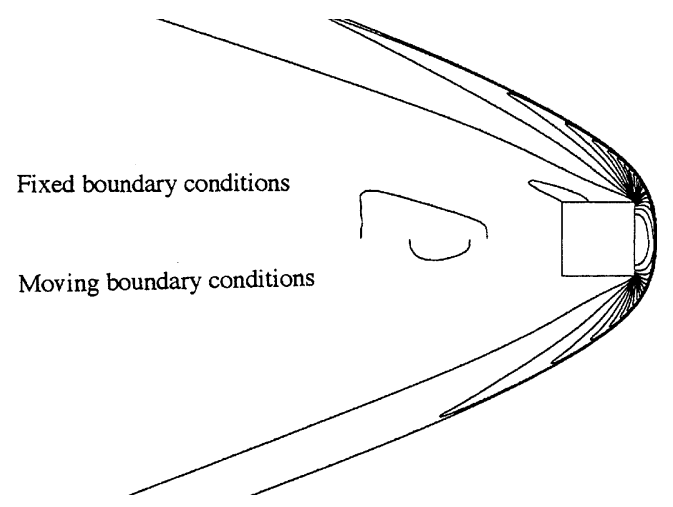

Fig. 3. Verification of moving boundary conditions, the projectile speed of $V_{\mathrm{p}}=1600 \mathrm{~m} / \mathrm{s}$.

position of each fringe matches well with each other with minor exceptions. In fact, the maximum shift in fringe position is less than one fourth of the distance between the two adjacent fringes. This maximum discrepancy means that errors in the integrated density are less than $4 \%$ of the initial density according to the fringe pattern analysis because one fringe shift corresponds to a density shift of $13.68 \%$ of the initial density in front of the transmitting shock wave. Considering the difficulty in timing numerical results to match exactly the experiment and some experimental uncertainty, such a level of accuracy is perfectly acceptable to confirm that the numerical simulations represents the physics occurring in the shock wave propagation.

\subsection{Verification of moving boundary conditions}

The second case for verification of the moving boundary conditions is a projectile flying in open space at a supersonic speed. Simulations were carried out by solving the governing equations first in the projectile-fixed coordinates with fixed boundary conditions, and then in the laboratoryfixed coordinates with moving boundary conditions. The flow field suffers the Galilean transformation but thermal state parameters remain identical. These results are presented with isobars in Fig. 3, where the result with the fixed boundaries is plotted in the upper half and that with the moving boundaries is in the lower half. Good agreement was observable from the stand-off distance of the bow shock wave and from the number of isobars in the flow field, except a slight shift of the position of the two corresponding isobars in the wake. In fact, the discrepancy of the stagnation pressure in front of the projectile is less than $7 \%$. Hence the reliability of the moving boundary conditions was well demonstrated.

\section{Results and discussion}

In the following discussion, a number of major wave dynamic processes will be identified, which interact with each other in different ways. The first process is the generation of the first blast wave resulting from the gas behind the precursor shock wave, which will disturb the flow it has passed. 
The second process is the second blast wave induced by the high pressure gas behind the projectile, including its generation, motion and interaction with the first blast wave. The third one is the instability development of contact surfaces and shear layers, which is induced by reflected shock waves and shock wave focusing. The last process of the moving shock wave interaction occurs later when the bow shock wave catches up with the precursor shock wave, from which a new bow shock wave develops and a contact surface follows.

\subsection{Case 3: the projectile moving at a Mach number of $M_{p}=2.0$}

The third case is the shocked flow induced by a projectile moving at a Mach number of $M_{p}=2.0$ and its numerical results are presented in a time sequence in Fig. 4. In this figure, isobars are plotted in the lower half of each frame and isopycnics in the upper half at the same time.

Fig. 4(a) shows the frame at $t=162 \mu \mathrm{s}$, when the projectile is just released from the shock tube and the precursor shock wave driven by the projectile has propagated into the big chamber. In this early frame, the precursor shock wave has transmitted into a sphere shape; a contact surface follows, which separates the gas being initially in the shock tube from that in ambient air; a secondary shock wave is generated due to locally developed supersonic flows, the rim of which sucks into the low-pressure core of the primary vortex ring; an under-expanded jet develops and the barrel shock is generated. Later, as shown in Fig. 4(b) at $t=230.5 \mu$ s, the precursor shock wave reflected from the rigid chamber wall and the reflected shock wave interacts with the contact surface. In Fig. 4(c) at $t=282.7 \mu$ s, the high pressure gas behind the projectile rushes out and the second blast wave, named after the transitional ballistic regime, develops. There is nothing that is observable in front of the projectile, because the projectile moves in the region where the particle velocity is approximately the same to the projectile speed. Meantime, the reflected shock interacts with the primary vortex ring and the distorted portion of the shock wave bends backward. Fig. $4(\mathrm{~d})$ at $t=338.2 \mu \mathrm{s}$ shows that a bow shock is driven in front of the projectile because the projectile speed is much higher than the particle velocity in front of it. A cylindrical shock wave with a curved front was observed beside the projectile in Fig. 4(e) at $t=442.7 \mu \mathrm{s}$, propagating in the radial direction. Actually, this is the leading shock wave of the second blast wave, but distorted in the jet flow region where flow velocity varies dramatically from the axis of symmetry. On the other hand, the contact surface and the shear layer are also disturbed by the shock wave in return.

The leading shock wave of the second blast wave overtakes the projectile from Fig. 4(d) and interacts with the bow shock wave in Fig. 4(e) and develops into a curved shock wave in Fig. 4(f) at $t=498.1 \mu$ s, clearly delineating the flow influenced by the projectile from that by the precursor shock wave. The reflection of the precursor shock wave from the rigid chamber wall transits from a regular reflection as shown in Fig. 4(b) to a Mach reflection in Fig. 4(d), in which a shear layer is clearly visible from the triple-point. The reflection of the curved shock occurs from Fig. 4(e) and the reflected shock wave interacts with the shear layer in Fig. $4(\mathrm{~g})$ at $t=561.8 \mu \mathrm{s}$, which results in intense shear layer instability in Fig. $4(\mathrm{~g})$ at $t=561.8 \mu \mathrm{s}$. Meanwhile, a new shear layer originating from the frontal edge of the projectile is generating because the projectile speed is much faster than the particle velocity around it. A shock wave focusing behind the projectile occurs from Fig. 4(d) and (e), which results from the secondary shock wave diffracting over the rear surface of the projectile as it moves through the shock wave. This becomes another source that will generate perturbations on the wake behind the projectile, as shown in Fig. 4(e) and (f). The curved shock 


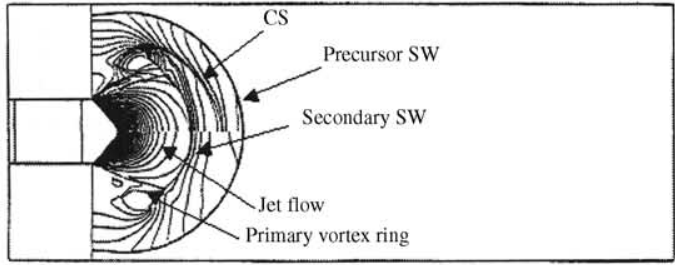

(a)

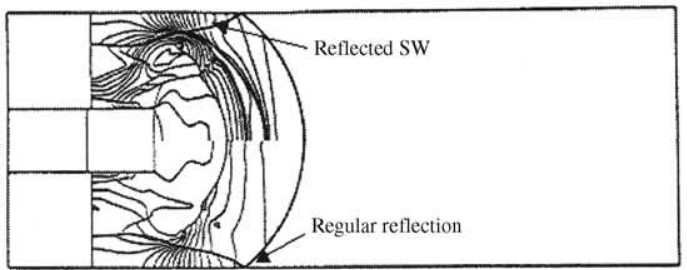

(b)

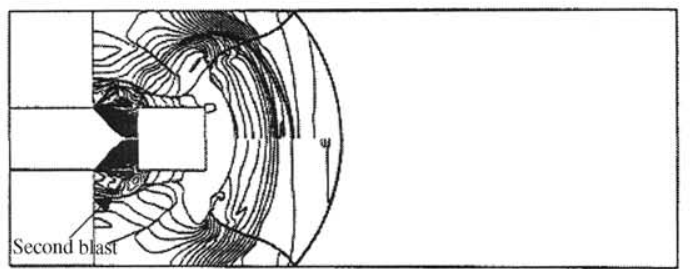

(c)

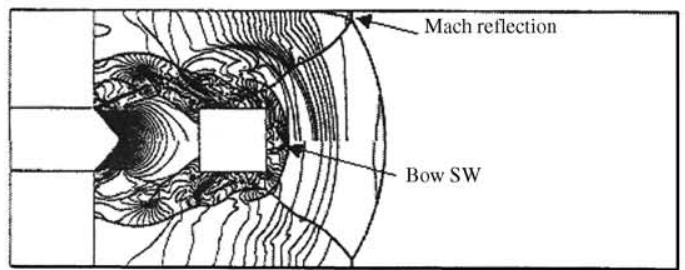

(d)

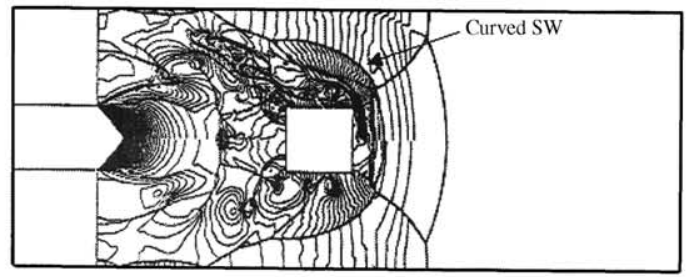

(e)

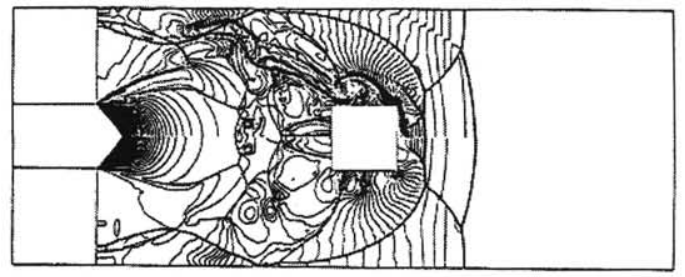

(f)

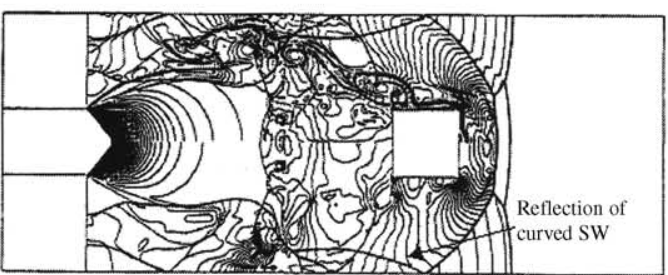

(g)

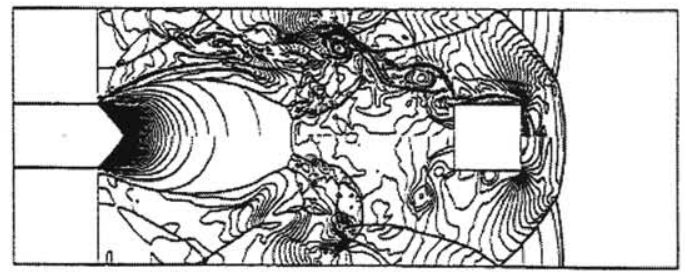

(h)

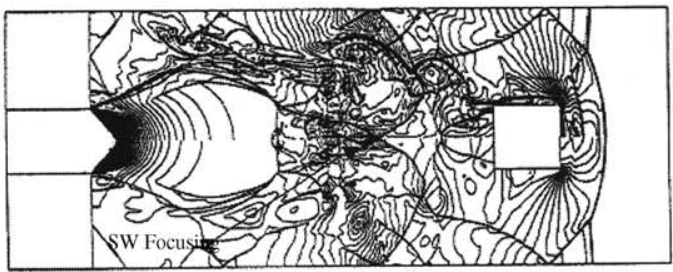

(i)

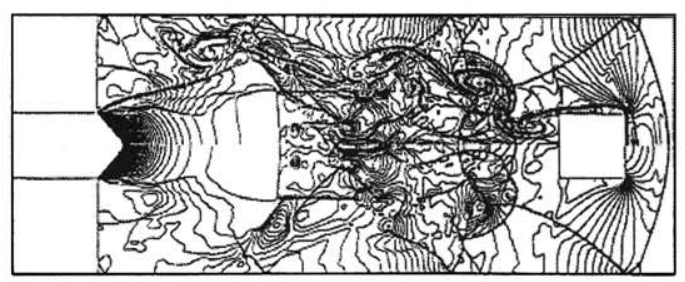

(j)

Fig. 4. A time sequence of isobars (lower) and isopycnics (upper) showing the shocked flow induced by the projectile moving at $M_{p}=2.0, \mathrm{CS}$ — contact surface; SW—shock wave; second blast—the second blast wave.

wave catches up with the precursor shock wave in Fig. 4(h) at $t=618.9 \mu \mathrm{s}$. This shock wave interaction leads to the the generation of a contact surface and a series of expansion waves. The expansion waves run away quickly and is the only contact surface observable in front of the 
projectile, as shown in Fig. 4(i) at $t=684.1 \mu$ s. A new precursor shock wave develops in Fig. 4(j) at $t=752.7 \mu \mathrm{s}$, and can be also defined as the new bow shock wave. Only after this stage, the flowfield around the projectile may be approximately treated as a steady state with the Galilean transformation.

By examining the flowfield between the Mach disk shock and the projectile at the frame shown in Fig. 4(h), it is found that a shock wave propagates toward the axis of symmetry, which results in the shock wave focusing in Fig. 4(i). Fig. 4(h) shows also that the reflected shock wave from the chamber wall interacts with the shear layer, which imposes more disturbances on the shear layer development and leads to its rolling up in Fig. 4(i). The effect of these interactions is observable in Fig. 4(j), where complex shock-wave phenomena appear behind the projectile and the wellshocked flow develops. It seems that turbulent flows observed in this region from experiments in many previous work are due to these wave dynamic processes. Although the present simulations do not reproduce correctly the flow state, but do provide valuable information for understanding on the mechanism of flow state transitions.

\subsection{Case 4: the projectile moving at a Mach number of $M_{p}=2.4$}

Numerical results of case 3 presented in the last section shows the development of the shocked flow and a number of major wave dynamics phenomena in it. The last case, the projectile moving at a higher Mach number of $M_{p}=2.4$, is carried out to examine the effect of the projectile speed on the shocked flow. The numerical results of case 4 are presented in Fig. 5 in a time sequence of pressure and density distributions. The same display style is adopted as is used in Fig. 4.

Generally speaking, wave dynamic processes occurring in case 4 appear to be similar to these being observable in case 3, for example, the first blast wave in Fig. 5(a) at $t=135 \mu$ s; the second blast wave behind the projectile in Fig. 5(b) at $t=227.5 \mu \mathrm{s}$; the development of the bow shock wave in Fig. 5(c) at $t=356.7 \mu \mathrm{s}$; the bow shock joining the leading shock wave of the second blast wave in Fig. 5(d) at $t=431.6 \mu$ s, from which a curved shock wave develops; the reflection of the curved shock wave sweeping over the shear layer and the contact surface in Fig. 5(e) at $t=466.9$ $\mu$ s to Fig. 5(h), which leads to intense flow instability; catching up of the bow shock wave with the precursor shock wave in Fig. 5(f) at $t=515.9 \mu \mathrm{s}$; instability of the shear layer from the projectile frontal edge in Fig. 5(g) at $t=567.6 \mu \mathrm{s}$; and a new bow shock generating in Fig. 5(h) at $t=635.6$ $\mu \mathrm{s}$. However, there are still some discrepancies observable, which result from the higher projectile speed. The first one is the shock cell in case 4 is wider and longer than in case 3 , which is concluded by comparing Fig. 5(c)-(h) with Fig. 4(e)-(j). This is because the jet flow associated with the second blast wave is more highly under-expanded due to the higher flow pressure behind the projectile in case 4 . The second is the stronger reflection of the precursor shock wave, which is identified from the contact surface originating from the triple-point in Fig. 5(c) and (d), which is more intense than in Fig. 4(e) and (f). The third is the stand-off distance of the precursor shock wave. It is shorter in Fig. 5(h), but longer in Fig. 4(j). This is because the new bow shock in case 4 is stronger due to the higher projectile speed. Another difference is the shock wave focusing as shown in the central area of Fig. 5(h), which is also more intense than in Fig. 4(i). In conclusion, the wave dynamics processes in case 4 did not change significantly as the projectile speed increases, but wave interactions appear more intense. It may imply that more vortices and turbulence will be induced. 


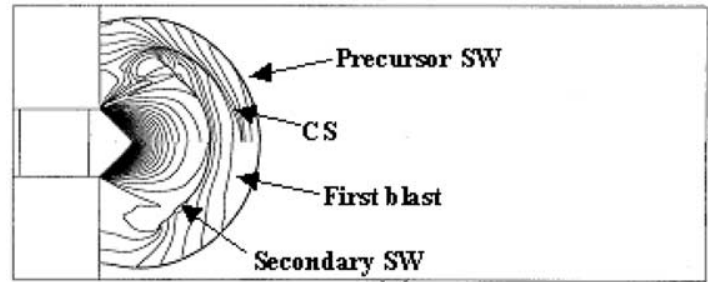

(a)

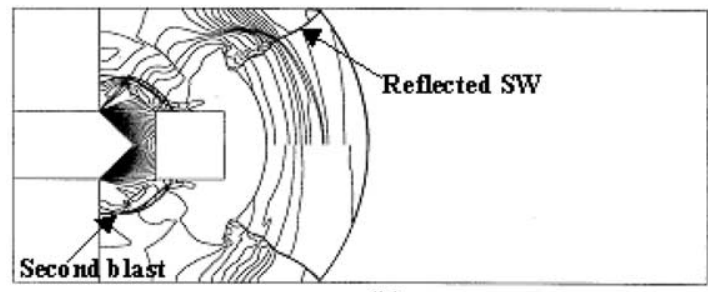

(b)

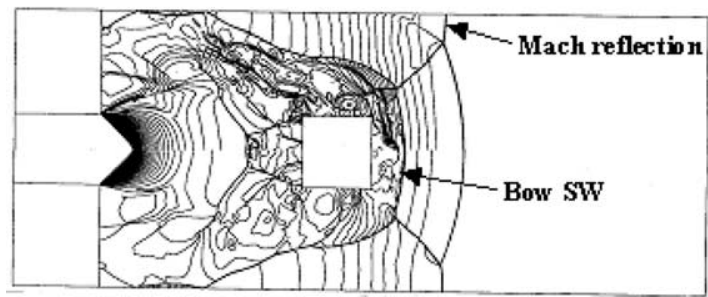

(c)

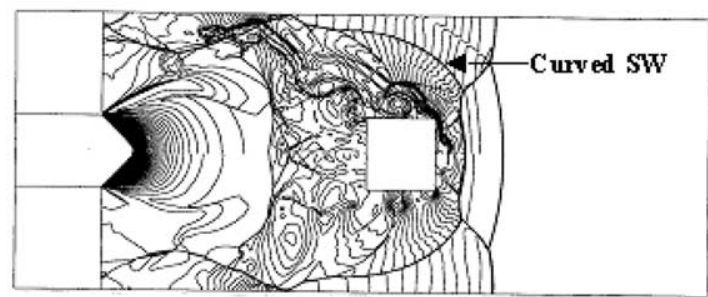

(d)

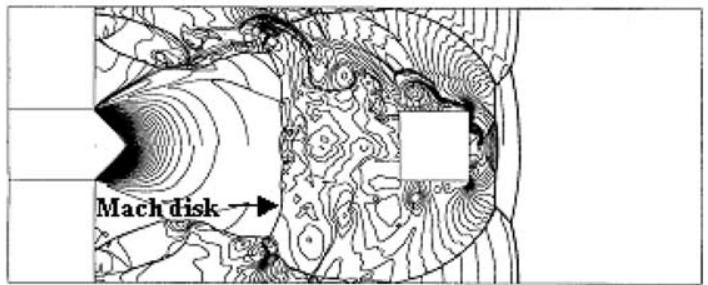

(e)

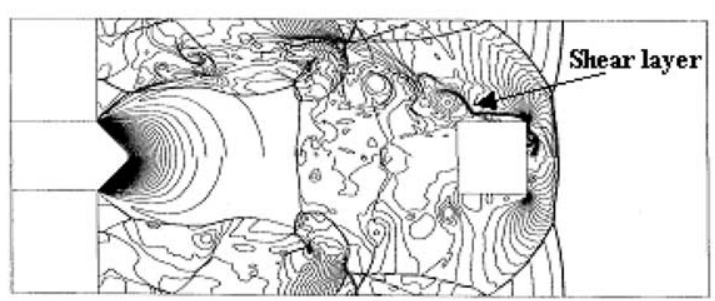

(f)

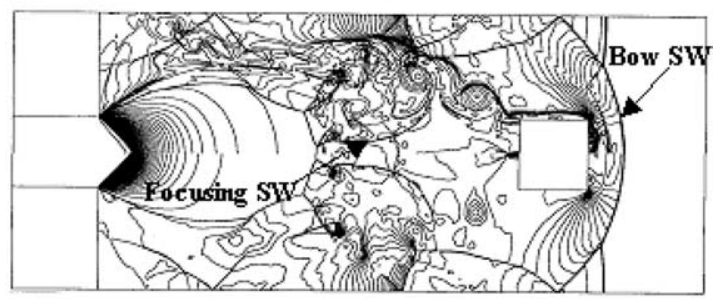

(g)

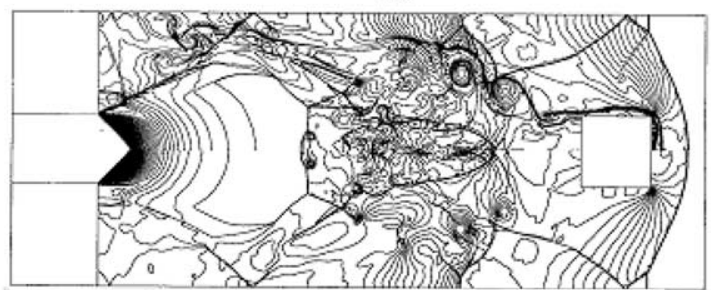

(h)

Fig. 5. A time sequence of isobars (lower) and isopycnics (upper) showing the shocked flow induced by the projectile moving at a Mach number of $M_{p}=2.4$. First blast—the first blast wave; bow SW—bow shock wave.

\subsection{Computational schlieren results}

Figs. 4 and 5 show complex shocked flows induced by a supersonic projectile moving from a shock tube into a big chamber. A question is that it is possible to visualize the wave dynamics processes with flow visualization techniques, for example, with schlieren method. With the algorithms introduced by Jiang et al. [12], computational schlieren results are created by integrating the cylindrical density distribution of the numerical simulations of case 3 , and presented in Fig. 6 in a time sequence. From this figure, the reflection of the precursor shock wave in Fig. 6(a) at $t=440.7 \mu \mathrm{s}$, the bow shock wave generation in Fig. 6(b) at $t=470.0 \mu \mathrm{s}$, catching up of the bow shock wave with the precursor shock wave in Fig. 6(c) at $t=603.8 \mu \mathrm{s}$, the development of the new 

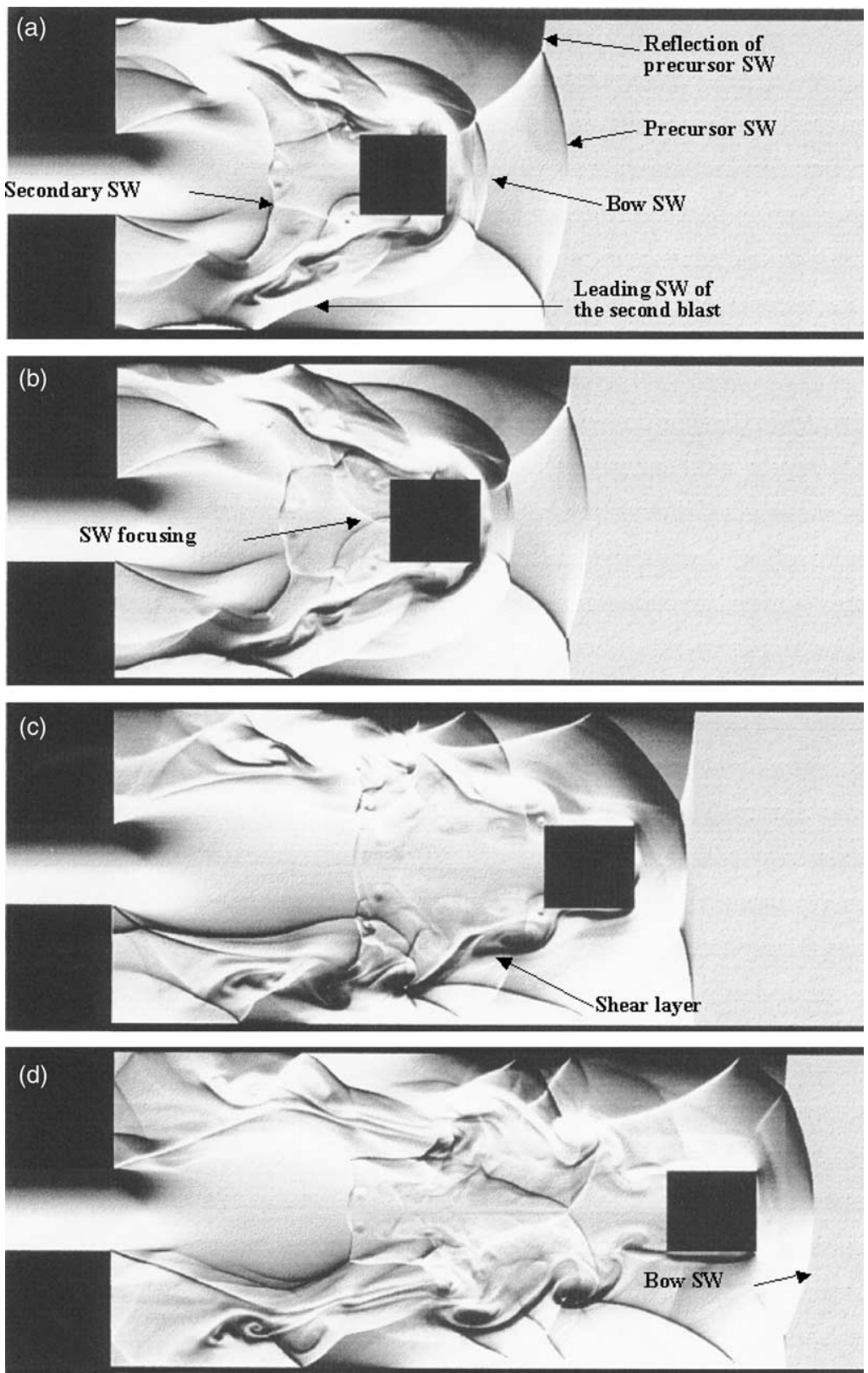

Fig. 6. A time sequence of numerical schlieren photos showing wave interactions in the shocked flow induced by the projectile moving at a Mach number of $M_{p}=2.0$.

bow shock wave in Fig. 6(d) at $t=708.3 \mu$ s, and the instability development of the contact surface and the shear layer from Fig. 6(b)-(d) while the reflected shock waves from the chamber wall are sweeping over them. All the wave phenomena are standing out clearly against the background 
flow, demonstrating vividly intensive wave interactions. As is well known, schlieren results include the effect of integrating three-dimensional density distribution along light path, some physics may be smeared. However, most of the wave dynamics phenomena being observable in Fig. 4 are also observable in Fig. 6, even can be more clearly identified. This is a helpful indication for investigating further the shocked flow by motivating the experimental study on the present case.

\section{Conclusions}

From the discussion in the above sections, the numerical investigation can be summarized as follows: The dispersion-controlled scheme implemented with the moving boundary conditions were well validated and the shocked flow induced by a supersonic projectile moving from a shock tube into a big chamber was simulated successfully. The shocked flow is characterized with several major wave dynamic processes: the generation of two blast waves; the reflection of the precursor shock wave and the leading shock wave of the second blast wave; the interaction among reflected shock waves, contact surfaces and shear layers; catching up of the bow shock with the precursor shock wave; and shock wave focusing behind the projectile. The wave dynamic processes do not change significantly as the projectile speed increases, but wave interactions do appear more intense. These wave dynamics processes and their interactions will induce vortices and turbulence in the wake behind the projectile, which agrees with previous experimental observations and is worth to be investigated further in future.

\section{References}

[1] Nusca MJ. Reacting flow simulation of transient, multi-stage ram accelerator operation and design studies. Proc Second Int Workshop on Ram Accelerators. University of Washington, Seattle, WA, USA, 1995.

[2] Hinkey Jr JB. An experimental and numerical investigation of the three-dimensional flow field about a ram accelerator projectile. Proc Second Int Workshop on Ram Accelerators. University of Washington, Seattle, WA, USA, 1995.

[3] Glass II. Shock waves and man. Toronto, Canada: The University of Toronto Press; 1974.

[4] Matsumura T, Funabashi S, Taitoh T, Takayama K. A holographic interferometric study of the axisymmetric supersonic flow around a cylindrical projectile. Rep Ins Fluid Sci Tohoku Univ 1993;5:89-97.

[5] Jiang Z, Takayama K. Numerical study on blast flowfields induced by supersonic projectiles discharged from shock tubes. Phys Fluids 1998;10(1):277-88.

[6] Taki S, Zhang C, Chang X. Numerical simulation of the unsteady processes in starting period of ram accelerator. Proc Third Int Workshop on Ram Accelerators, Sendai, Japan, 16-18 July 1997. p. 215-21.

[7] Henner M, Giraud M, Legender JF, Berner C. CFD computations of steady and non-reactive flow around finguided projectiles. Proc Third Int Workshop on Ram Accelerators. Sendai, Japan, 16-18 July 1997. p. 215-21.

[8] Oswatitsch K. Intermediate Ballistics, DVL Rept. 358, June 1964, Deutschen Versuchsanstalt fur Luft-und Raumfahrt, Aachen, Germany.

[9] Jiang Z. Study on the finite difference theory and numerical methods of weak solution problems. Doctoral Thesis, Peking University, Beijing, China, July 1993.

[10] Jiang Z, Takayama K, Chen YS. Dispersion conditions for non-oscillatory shock capturing schemes and its applications. Computat Fluid Dyn J 1995;2(4):137-50.

[11] Steger JF, Warming RF. Flux vector splitting of the inviscid gasdynamic equations with applications to finite difference methods. J Computat Phys 1981;40:263-93. 
[12] Jiang Z, Takayama K. An investigation into the validation of numerical solutions of complex flowfields. J Computat Phys 1999;151:479-87.

[13] Jiang Z, Chen YS, Kuwahara K. Approximate non-reflecting inflow-outflow boundary conditions for the calculation of Navier-Stokes equations in internal flows. Acta Mech Sinica 1993;4(9):289-97.

[14] Schmidt E, Shear D. Optical measurements of muzzle blast. AIAA J 1975;13:1086.

[15] Jiang Z, Matsuoka K, Sasoh A, Takayama K. Numerical and experimental investigation of wave dynamic processes in high-speed train/tunnels. Acta Mech Sinica 2002;18(3):209-26. 